\title{
Approach to Abnormal Uterine Bleeding in Adolescents
}

\author{
D Cenk Yaşa, (D) Funda Güngör Uğurlucan \\ istanbul University, Istanbul Faculty of Medicine, Department of Obstetrics and Gynecology, Istanbul, Turkey
}

\begin{abstract}
This article reviews the current understanding and management of abnormal uterine bleeding (AUB) in adolescents. It is hoped that this review will provide readers with an approach to the evaluation and treatment of mild to severe uterine bleeding. AUB is a common problem which has significantly adverse effects on an affected adolescent's quality of life. The most common underlying condition in AUB in adolescence is anovulation. During the evaluation, pregnancy, trauma and sexually transmitted diseases must be ruled out, regardless of history. It should be kept in mind that AUB during this period may be the first sign of underlying bleeding disorders. Although observation is sufficient in the mild form of AUB, at the other end of the spectrum life-threatening bleeding may necessitate the use of high doses of combined oral contraceptives, intravenous estrogen and/or interventional procedures.
\end{abstract}

Keywords: Abnormal uterine bleeding, adolescents, heavy menstrual bleeding, oral contraceptive pills, coagulopathy

\section{Introduction}

Adolescents have frequent menstrual problems such as irregular menses, painful cycles and prolonged or heavy menstrual bleeding (HMB). Abnormal uterine bleeding (AUB) is defined as bleeding from uterine corpus that is abnormal in duration, volume, frequency and/or regularity. Due to immaturity of the hypothalamic-pituitaryovarian (HPO) axis, AUB is common in adolescents $(1,2)$. Furthermore, inherited or acquired bleeding diathesis may further intensify the existing hormonal imbalance and increase morbidity of the underlying condition. In addition to these problems, hyperprolactinemia, thyroid disorders and polycystic ovary syndrome (PCOS) are the common underlying endocrine disorders. AUB decreases quality of life, affects school attendance and limits sports and social activity participation (3). Although the management of this problem has evolved over time, the most important goal remains to alleviate the anxiety of both affected girls and their families and to identify the underlying medical conditions that may have chronic health effects for these girls. In this paper, the most common causes of AUB will be discussed and current management will be reviewed.

\section{Normal Menstrual Cycle in Adolescents and Classification of AUB}

Although the age of onset of puberty has tended to decrease over the past few decades, the age of menarche has remained constant at 12-13 years (4). At present, more than $90 \%$ of girls are menstruating before age of 14 years. Menarche is generally considered as anovulatory bleeding. The time required for HPO axis maturation following menarche, which is thought to result in ovulatory cycles and subsequent regular bleeding, varies between six months and three years. Due to ovulatory dysfunction, in the following months after menarche, irregular and unpredictable, heavy and prolonged, and, rarely, skipped menses for less than three months may occur (5). Thus perception of "normal" menstrual cycle may vary in these girls and their families. As in adults, menstrual cycles are between 21 and 34 days, last for seven days or fewer, with an average blood loss of 30-40 mL leading to 3-6 pads or tampon usage per day (6). HMB is the most common form of AUB and is defined as excessive menstrual blood loss that interferes with a woman's physical, social, emotional or material quality of life (7). Some additional signs of HMB include changing pad or tampon more often than every one to two hours, use 
of double hygiene protection, frequent soiling of clothes or bed sheets and blood clots more than one inch $(2.54 \mathrm{~cm})$ in diameter (8). The causes of HMB may be classified using the Polyp, Adenomyosis, Leiomyoma, Malignancy-Coagulopathy, Ovulatory dysfunction, Endometrial, Iatrogenic and Not yet classified (PALM-COEIN) classification which is divided into structural causes including PALM and hyperplasia and nonstructural causes which include COEIN (9). The structural causes of HMB are rarely seen in the adolescent age group.

AUB might also be classified as acute or chronic. Acute AUB refers to an episode of heavy bleeding which is sufficient in quantity to require immediate intervention to prevent further blood loss. Abnormalities in quantity, regularity and/or timing in the last six months may all be defined as chronic AUB (10). Usually, chronic menstrual bleeding that exceeds $80 \mathrm{~mL}$ will result in anemia.

\section{Clinical Evaluation}

AUB in adolescents is a challenging and often neglected problem. Cycle to cycle variability, differences in menstrual hygiene, the wide variety of menstrual hygiene pads or tampons available, inconsistency in giving information about menstrual regularity and bleeding amounts make initial assessment of AUB even more difficult in these girls (11). Patients and their families may not know what is "normal" and patients may not inform their families about menstrual irregularities. In addition, most bleeding disorders may not be as obvious until menarche. Although during this period, AUB may occur more frequently due to anovulation, bleeding disorders may also accompany this condition.

\section{Differential Diagnosis}

In these girls, clinicians should evaluate the features of the menstrual cycle like a vital sign (12). An accurate history of patient's cycles is the main issue for diagnosis, to determine if her experiences are normal or abnormal. The onset of menarche, cycle length, variability over time and the amount of menstrual bleeding should be evaluated. After providing a suitable conversation environment, sexual activity should be questioned. Pregnancy and its related complications should also be part of the initial investigation in girls presenting with AUB. Although AUB is often caused by anovulatory cycles, severe bleeding may be the first sign of an underlying condition and this may be a diagnosis of exclusion. PCOS, another cause of anovulatory cycles, should be kept in mind as a common underlying etiology of AUB, since it can easily be missed in this age group (13). Excessive bleeding during menarche can usually indicate an underlying bleeding disorder, while regular but excessive bleeding may also be indicative of bleeding disorders. Von Willebrand disease, platelet function defects, thrombocytopenia and clotting factor deficiencies are the most common bleeding disorders in adolescent girls that present with HMB. Up to $36 \%$ of adolescents with AUB may have an underlying coagulopathy (14). Using a screening tool for underlying bleeding diathesis in adolescents with AUB can assist the physician.

Initial screening for an underlying disorder of hemostasis in patients with excessive menstrual bleeding should be structured by the medical history. A positive screening result comprises the following circumstances:

- Heavy menstrual bleeding since menarche

- One of the following conditions:

- Postpartum hemorrhage,

- Surgery-related bleeding,

- Bleeding associated with dental work.

- Two or more of the following conditions:

- Bruising, one to two times per month,

- Epistaxis, one to two times per month,

- Frequent gum bleeding,

- Family history of bleeding symptoms.

Patients with a positive screening test should be considered for further evaluation, including consultation with a hematologist and testing for von Willebrand factor and ristocetin cofactor.

In intermenstrual bleeding, cervicitis and hormonal contraception may be implicated. In adolescents who do not respond to standard medical therapy, structural causes of bleeding should be excluded. Table 1 for common causes for AUB in adolescents

\section{Physical Examination}

When adolescents present with acute AUB, physical examination should focus on signs of acute blood loss and the etiology of bleeding. While tachycardia and orthostatic hypotension may be the only signs of severe anemia, it should be kept in mind that young patients will not present with clinical signs, despite severe anemia. While the presence of bruises and petechiae on the skin may indicate an underlying coagulation disorders, pallor may be seen due to anemia. In adolescents who are sexually active, trauma, foreign body, structural causes and pelvic inflammatory diseases can be investigated by pelvic and bimanual examination. 


\section{Laboratory Evaluation and Imaging}

Initial evaluation of adolescents presenting with acute AUB should include screening for pregnancy, anemia, bleeding disorders, iron deficiency and thyroid disease (15). Complete blood count, blood type, cross match and pregnancy test should be first line tests. In addition, partial thromboplastin time, prothrombin time, activated partial thromboplastin time and fibrinogen level are the initial evaluation for disorders of hemostasis. All adolescents with abnormal initial test or positive screening results for disorders of hemostasis should be evaluated by assessment of von Willebrand-ristocetin cofactor activity, von Willebrand antigen and factor VIII for diagnosis of von Willebrand disease and other coagulopathies (16).

Since exogenous estrogen use may increase von Willebrand Factor concentrations into the normal range, it is necessary to perform the test either before starting hormone treatment or seven days after the end of treatment to prevent false negative results (17). If patient's history or physical examination findings are suggestive of PCOS, testosterone (free/total), DHEAS and prolactin should be evaluated.

\begin{tabular}{ll}
\hline $\begin{array}{l}\text { Table 1. Differential diagnosis of abnormal uterine } \\
\text { bleeding in adolescents }\end{array}$ & \\
\hline Endocrine & Medications \\
Anovulatory bleeding & Anticoagulants \\
Polycystic ovary syndrome & $\begin{array}{l}\text { Depot medroxyprogesterone } \\
\text { implants }\end{array}$ \\
Thyroid disorders & Intrauterine devices \\
Hyperprolactinemia & Trauma \\
Bleeding disorders & Foreign bodies \\
Von Willebrand disease & \\
Platelet dysfunction & \\
Thrombocytopenia & \\
Clotting factor deficiency & \\
Pregnancy & \\
Abortion & \\
Ectopic pregnancy & \\
First trimester bleeding & \\
Gestational trophoblastic & \\
disease & \\
Infections \\
Cervicitis \\
Endometritis \\
Sexually transmitted \\
disease \\
Uterine pathologies \\
Polyp \\
Leiomyoma \\
Adenomyosis
\end{tabular}

Sexually active adolescents should be screened for Neisseria gonorhhea and Chylamidia trachomatis infections with nucleic acid amplification tests.

Routine pelvic imaging is considered unnecessary since structural etiologies are rarely seen in this group. However, in the girls who do not respond to initial treatment, transabdominal ultrasonography may be more appropriate than transvaginal ultrasonography.

\section{Management}

Most adolescents need outpatient management and reassurance that their menstrual cycles would become cyclic and ovulatory over time. However, treatment is required when AUB causes anemia or impairs quality of life (18). In these girls, the first line treatment is generally medical. Surgical options should be reserved for girls who can't be managed by medical treatment. Acute AUB patients who are clinically unstable, have active bleeding or severe anemia should be hospitalized for management (19). A clinical decision should be made regarding intravenous crystalloid and blood or blood product transfusions, hormone treatment, and iron replacement, according to the severity of bleeding, clinical condition of the patient, hemodynamic stability and the underlying medical problem. If an underlying cause can be identified, appropriate specific treatment should be given.

\section{Management of Girls with Acute Bleeding}

Girls with active, profuse, heavy bleeding ( $>1$ pad per hour), presence of vital signs in conjunction with evidence of hypovolemia, orthostatic hypotension or hemoglobin (Hb) concentration $<8 \mathrm{gr} / \mathrm{dL}$ due to bleeding are accepted as having severe AUB and should be hospitalized. If patients can tolerate oral intake, and in the absence of contraindications of estrogen treatment, monophasic combined oral contraceptive pills (OCP) containing 30-50 mcg ethinyl estradiol, can be used every 6-8 hours until bleeding diminishes, then taper to two and then one pill daily (19). If bleeding does not decrease after the first two doses of OCP or patients are not able to take oral hormone treatment, intravenous $25 \mathrm{mg}$ conjugated estrogen every 4-6 hours should be considered until bleeding ceases (20). Most adolescents respond quickly to hormone treatment and iron supplementation and also tolerate anemia better than adults. Therefore, blood transfusion should be avoided as far as possible until the occurrence of hemodynamic instability or the presence of symptoms of severe anemia. There is no established $\mathrm{Hb}$ concentration for transfusion requirement. Furthermore, if transfusion is to be performed, one unit of packed red cell should be given and the need 
of further transfusion should be re-evaluated according to the subsequent status of the patient. Platelet transfusion is rarely required except in cases of severe thrombocytopenia or platelet disorder (21). In addition, when a deficiency of coagulation factors has been identified, clotting factors from plasma derived concentrate or recombinant agents might be needed (22). After cessation of bleeding, transition to maintenance treatment is required. High dose estrogen treatment can induce nausea and vomiting so anti-emetic agents should be begun in a prophylactic manner. If bleeding cannot be managed by these measures within 24-48 hours, consultation with a hematologist should be considered. During the maintenance period, continuous OCP (active pills only) which contain 30-50 mcg ethinyl estradiol with norgestrel or levonorgestrel (LNG), should be continued until $\mathrm{Hb}$ concentrations increase, or for longer in the presence of underlying bleeding disorders. In girls with a contraindication to estrogen-containing regimens, progesterone in the form of oral medroxyprogesterone acetate at a dose of 10-20 mg every 6-12 hours or oral norethindrone acetate at a dose of 5-10 mg every six hours are effective. Again, tapering of dose is begun after bleeding diminishes. Once the patient's bleeding ceases and $\mathrm{Hb}$ level is stabilized, the patient could be discharged from hospital after toleration for oral therapy is established. During the maintenance period, oral iron supplementation, along with dietary counseling to increase iron intake, should be given until iron stores are restored as indicated by a normal ferritin concentration. An oral dose of iron of 60-120 mg per day is recommended. Recently, evidence has been presented which suggested that daily single dose use is better than multiple daily doses (23). If there are concerns about oral iron intake, intravenous iron treatment may be initiated for these girls during the hospitalization period. In girls who have menstrual bleeding under control, iron support is usually sufficient for 3-6 months. Complete blood count and iron studies should be performed to determine when to terminate the supplementation.

Although it is known that nonsteroidal anti-inflammatory drugs (NSAIDs) decrease menstrual bleeding in premenopausal women, NSAIDs should not be prescribed to these girls because this therapy may exacerbate AUB due to underlying bleeding disorders. Tranexamic acid is an antifibrinolytic agent that has been shown to be as effective in decreasing menstrual blood loss as OCP and improved the quality of life in adolescents (24). Concomitant use of tranexamic acid and OCP is contraindicated according to drug information because there is a hypothetical increased risk of thrombosis. However, the increased risk of thrombosis with combined use has not been demonstrated by long-term clinical experiences (25). Thus, despite this risk, OCP and tranexamic acid combination has been used in patients who fail to respond to treatment with OCP alone. The recommended dose of tranexamic acid is $1300 \mathrm{mg}$ orally or $10 \mathrm{mg} / \mathrm{kg}$ intravenously (maximum $600 \mathrm{mg} / \mathrm{dose}$ ) three times daily for up to five days (26). Aminocaproic acid, another anti-fibrinolytic agent, is both less effective and has more side effects (27). Desmopressin is a synthetic analogue of the vasopressin. It increases concentrations of von Willebrand Factor and Factor VIII. It also causes platelet adhesion. It is commonly used in type 1 von Willebrand disease, hemophilia and platelet function defects in the form of a nasal spray (28).

First-line medical management may fail to result in cessation of bleeding in some patients who may require interventional procedures and further evaluation. Even in cases of lifethreatening bleeding, procedures such as uterine artery embolization, endometrial ablation and hysterectomy should not be performed, as these treatment modalities may cause future infertility. In these patients, pelvic ultrasonography and pelvic examination under general anesthesia might provide further evidence for clinical decision making. If the presence of clot or decidual cast is demonstrated by ultrasonography, uterine evacuation or suction curettage might be appropriate. An alternative intervention to stop bleeding may be intrauterine balloon insertion. Studies of balloon insertion, especially in women with postpartum bleeding, have reported efficacy in controlling bleeding. A Foley catheter is a low-risk, low-cost, and readily accessible intrauterine balloon to consider for young girls and adolescents (29). Since the uterine volume of these girls is small, the amount of inflation needed for effective tamponing is judged by the amount of wall resistance felt. The balloon may remain in situ for 12-24 hours while other treatments are given. After the bleeding stops, the balloon of the Foley catheter is gradually and carefully emptied and completely withdrawn. There is a risk of uterine perforation, endometrial damage and infection risk with this method (30). Prophylactic antibiotics should be given, as long as the balloon remains, for prevention of infection.

\section{Management of Girls with Mild or Moderate Bleeding}

Girls with light or mild bleeding, indicated by normal $\mathrm{Hb}$ concentrations, should be reassured that observation is sufficient, unless there is an impairment of quality of life. NSAIDs can be used to reduce the amount of bleeding. If bleeding persists or becomes more severe, re-evaluation of the patient is required. If the $\mathrm{Hb}$ concentrations of these girls are found to be in the 10-12 gr/dL range, observation or 
OCP are valuable therapeutic options and $60 \mathrm{mg}$ daily iron treatment should be commenced. If hormonal therapy is chosen, monophasic OCP, with 30-50 mcg ethinyl estradiol content, can be used every 8-12 hours until bleeding slows, then the therapy should be tapered to one pill daily over the course of a few days and therapy should be continued for at lesat 21 days.

In the presence of moderate bleeding or $\mathrm{Hb}$ concentration in the range 8-10 $\mathrm{gr} / \mathrm{dL}$, oral contraceptive treatment should be initiated as described above and continued until the $\mathrm{Hb}$ concentration is above $12 \mathrm{gr} / \mathrm{dL}$ with at least six months of iron supplementation. In the presence of a contraindication to estrogen therapy or alternative treatment in adolescents with anemia, progesterone therapy can be an option. Available progesterone therapies are oral medroxyprogesterone acetate (10 mg/day), micronized oral progesterone (200 mg/day) or norethindrone acetate (2.5-5 $\mathrm{mg} /$ day), which should be given for 12 days in every cycle (31).

\section{Long-term Management of Girls with Bleeding Disorders}

After acute menstrual bleeding ceases, these girls require treatment for long-term bleeding control. In addition to diet optimization and iron supplementation, hormonal treatments are used. Hormonal treatments include OCP, oral, injectable and implantable progesterone and the LNG-releasing intrauterine device (LNG-IUD). For OCP, continuous or extended-cycle regimes are recommended for stabilization of the endometrium. Combinations of 30-50 mcg of ethinyl estradiol and levonorgestrel or norgestrel are more effective in reducing bleeding than in low-dose and new generation progesterone-containing preparations. Depot medroxyprogesterone acetate is also used for long-term bleeding control. Since there is a risk of hematoma with intramuscular administration, subcutaneous injection is recommended. To reduce the likelihood of initial breakthrough bleeding, therapy is applied more frequently than the usual 12-week cycle (32). Since daily, monthly and quarterly use of some formulas can be difficult for adolescents the LNG-IUD may be preferred. The LNG-IUD is active for up to five years after being placed in the uterine cavity. Additional benefits of the LNG-IUD are highly effective contraception, higher continuation rates and higher satisfaction rates when used for bleeding control compared with OCP in an adolescent population (33). In adolescents with bleeding disorders, the LNG-IUD has been demonstrated to be effective in controlling menstrual bleeding $(34,35)$. Since breakthrough bleeding caused by etonorgestrel implants is a common side effect, it is not usually used for the treatment of AUB.

\section{Conclusion}

AUB in adolescents may be acceptable at the beginning of the reproductive age when menstrual cycle regularity is not established, or may be the first sign of a severe underlying bleeding disorder. Girls with AUB should be evaluated with care and a wide differential diagnosis should be borne in mind. Medical therapy is usually an effective and sufficient treatment. Generally, adolescents respond well to therapy. Hematology consultation, imaging methods and clinical intervention should be considered in patients who do not respond to treatment.

\section{Ethics}

Peer-review: Internally peer-reviewed.

\section{Authorship Contributions}

Surgical and Medical Practices: Cenk Yaşa, Funda Güngör Uğurlucan, Concept: Cenk Yaşa, Funda Güngör Uğurlucan, Design: Cenk Yaşa, Funda Güngör Uğurlucan, Data Collection or Processing: Cenk Yaşa, Funda Güngör Uğurlucan, Analysis or Interpretation: Funda Güngör Uğurlucan, Literature Search: Cenk Yaşa, Writing: Cenk Yaşa.

Financial Disclosure: The authors declared that this study received no financial support.

\section{References}

1. Gray SH, Emans SJ. Abnormal vaginal bleeding in adolescents. Pediatr Rev 2007;28:175-182.

2. Benjamins LJ. Practice guideline: evaluation and management of vaginal bleeding in adolescents. J Pediatr Health Care 2009;23:189193.

3. Nur Azurah AG, Sanci L, Moore E, Groover S. The quality of life of adolescents with menstrual problems. J Pediatr Adolesc Gynecol 2013;26:102-108

4. Finer LB, Philbin JM. Trends in ages at key reproductive transitions in the United States, 1951-2010. Womens Health Issues 2014;24:271 279. Epub 2014 Apr 12

5. Jamieson MA. Disorders of menstruation in adolescent girls. Pediatr Clin North Am 2015;62:943-961. Epub 2015 Jun 10

6. Bennett AR, Gray SH. What to do when she's bleeding through: the recognition, evaluation and management of abnormal uterine bleeding in adolescents. Curr Opin Pediatr 2014;26:413-419.

7. National Institute for Health Institute for Health and Care Excellence. Heavy Menstrual Bleeding: assessment and management. London, 2007

8. Haamid F, Sass AE, Dietrich JE. Heavy menstrual bleeding in adolescents. J Pediatr Adolesc Gynecol 2017;30:335-340. Epub 2017 Jan 17 
9. Munro MG, Critchley HO, Broder MS, Fraser IS; FIGO Working Group on Menstrual Disorders. FIGO classification system (PALM-COEIN) for causes of abnormal uterine bleeding in nongravid women of reproductive age. Int J Gynaecol Obstet 2011;113:3-13. Epub 2011 Feb 22

10. American College of Obstetricians and Gynecologists. ACOG Committee Opinion No. 557: Management of acute abnormal uterine bleeding in nonpregnant reproductive-aged women. Obstet Gynecol 2013;121:891-896.

11. Sanchez J, Andrabi S, Bercaw JL, Dietrich JE. Quantifying the PBAC in a pediatric and adolescent gynecology population. Pediatr Hematol Oncol 2012;29:479-484.

12. No authors listed. ACOG Committee Opinion No. 651: Menstruation in girls and adolescents: using menstrual cycle as a vital sign. Obstet Gynecol 2015;126:143-146.

13. Masylyanskaya S, Talib HJ, Northridge JL, Jacobs AM, Coble C, Coupey SM. Polycystic Ovary Syndrome: An Under-recognized Cause of Abnormal Bleeding in adolescents admitted to children's hospital. J Pediatr Adolesc Gynecol 2017;30:349-355. Epub 2016 Nov 27

14. Deligeoroglu E, Karountzos V. Abnormal Uterine Bleeding including coagulopathies and other menstrual disorders. Best Pract Res Clin Obstet Gynaecol 2018;48:51-61. Epub 2017 Oct 9

15. Zia A, Rajpurkar M. Challenges of diagnosing and managing the adolescent with heavy menstrual bleeding. Thromb Res 2016;143:91100. Epub 2016 May 3

16. Committee on Practice Bulletins-Gynecology. Practice bulletin no. 128: Diagnosis of abnormal uterine bleeding in reproductive-aged women. Obstet Gynecol 2012;120:197-206.

17. Beller FK, Ebert C. Effects of oral contraceptives on blood coagulation. A review. Obstet Gynecol Surv 1985;40:425-436.

18. Kaunitz AM. Abnormal uterine bleeding in reproductive-age women. JAMA 2019;321:2126-2127.

19. No authors listed. Screening and Management of Bleeding Disorders in Adolescents With Heavy Menstrual Bleeding: ACOG COMMITTEE OPINION, Number 785. Obstet Gynecol 2019;134:71-83.

20. Devore GR, Owens O, Kase N. Use of intravenous premarin in the treatment of dysfunctional bleeding: a double-blind randomized controlled study. Obstet Gynecol 1982;59:285-291.

21. Rajpurkar M, O’Brien SH, Haamid FW, Cooper DL, Gunawardena S, Chitlur M. Heavy menstrual bleeding as a common presenting symptom of rare platelet disorders: illustrative case examples. J Pediatr Adloesc Gynecol 2016;29:537-541. Epub 2016 Feb 11

22. Kulkarni R. Improving care and treatment options for women and girls with bleeding disorders. Eur J Haematol 2015;95(Suppl 81):2-10.
23. Stoffel NU, Cercamondi CI, Brittenham G, Zeder C, Geurts-Moespot AJ, Swinkels DW, Moretti D, Zimmermann MB. Iron absorbtion from oral iron supplements given on consecutive versus alternate days and as single morning doses versus twice-daily split dosing in iron-depleted women: two open-label, randomized controlled trials. Lancet Haematol 2017;4:524-533. Epub 2017 Oct 9

24. Srivaths LV, Dietrich JE, Yee DL, Sangi-Haghpeykar H, Mahoney D Jr. Oral tranexamic acid versus combined oral contraceptives for adolescent heavy menstrual bleeding: a pilot study. J Pediatr Adolesc Gynecol 2015;28:254-257. Epub 2014 Dec 29

25. Thorne JG, James PD, Reid RL. Heavy menstrual bleeding: is tranexamic acid a safe adjunct to combined hormonal contraception? Contraception 2018;98:1-3. Epub 2018 Mar 26

26. Lukes AS, Moore KA, Muse KN. Tranexamic acid treatment for heavy menstrual bleeding: a randomized controlled trial. Obstet Gynecol 2006;108:924-929.

27. Levy JH. Hemostatic agents. Transfusions 2004;44(12 Suppl):58-62.

28. Edlund M, Blomback M, Fried G. Desmopressin in the treatment of menorrhagia and no common coagulation factor deficiency but with prolonged bleeding time. Blood Coagul Fibrinolysis 2002;13:225-231.

29. Stanley J, Adeyemi-Fowode O. Intrauterine foley balloon catheter to manage heavy menstrual bleeding in a perimenarchal 10-year-old girl. Obstet Gynecol 2019;134:77-80.

30. Hamani Y, Ben-Shachar I, Kalish Y, Porat S. Intrauterine balloon tamponade as a treatment for immune thrombocytopenic purpurainduced severe uterine bleeding. Fertil Steril 2010;94:2769. Epub 2010 Jun 9

31. Elmaogulları S, Aycan Z. Abnormal uterine bleeding in adolescents. J Clin Res Pediatr Endocrinol 2018;10:191-197. Epub 2018 Feb 28

32. Moon LM, Perez-Milicua G, Dietrich JE. Evaluation and management of heavy menstrual bleeding in adolescents. Curr Opin Obstet Gynecol 2017;29:328-336.

33. Committee on Adolescent Health Care. Committee Opinion No. 710. Counseling adolescents about contraception. Obstet Gynecol 2017;130:74-80.

34. Chi C, Huq Fy, Kadir RA. Levonorgestrel-releasing intrauterine system for the management of heavy menstrual bleeding in women with inherited bleeding disorders: long-term follow-up. Contraception 2011;83:242-247. Epub 2010 Aug 30

35. Silva CD, Geraldes F, Silva IS. Levonorgestrel intrauterine system as a treatment option for severe menorrhagia in adolescent with type III von Willebrand disease. BMJ Case Rep 2013:2013. 